\title{
Critical role of lay health cultural brokers in promoting the health of immigrants and refugees: A case study in the United States of America
}

\author{
Jerono P. Rotich ${ }^{1}$ \\ Adem Kaya $^{2}$
}

\begin{abstract}
The United States of America, a home to immigrants and refugees from various cultures and corners of the world continues to encounter waves of mass immigration. Some immigrated due to well-founded fears of persecution (i.e. religious, political, race, or social group) or economic hardships. Others immigrated to, reunite with family members, seek economic and education opportunities, and better standards of living. Notwithstanding their channels of admission or entry and their pivotal role in enriching the culture and the economy of the United States of America, many confront several health and lifestyle related challenges as they acculturate and integrate into the mainstream of American life and culture. Although many individuals and organizations have created numerous programs and activities to help ease these difficulties, minimal documentation is available on the involvement and engagement of the non-traditional work force, such as the lay health cultural brokers.

The purpose of this case study was to examine the role of lay health cultural brokers in promoting the health and acculturation of immigrants and refugees in the United States of America.

Methods: The participants for this qualitative case study were 10 lay health cultural brokers $(5$ male and 5 female) from different countries and cultures. The qualitative data was collected through focus group and one-on-one in-depth interviews.

Results: The findings indicated that the lay health cultural brokers played a critical role in helping immigrants and refugees acculturate and integrate into the American health care system. They served as interpreters, translators, system navigators, resource guides, educators and mentors among others.

Conclusion: The lay health cultural brokers provide essential cultural bridges and linkages that help to reduce the gaps that exist between the immigrants and refugees community and the American health care system.
\end{abstract}

Keywords: Immigrants; Refugees; lay health cultural brokers; Immigrant health; Health and acculturation; Lay health advisors; Immigrant Health access.

\footnotetext{
${ }^{1}$ Assoc. Prof., North Carolina Agricultural \& Technical State University, Department of Human Performance and Leisure Studies, USA, jprotich@ncat.edu

${ }^{2}$ Champions Wrestling School, Charlotte, USA, adem332@gmail.com
} 
Rotich, J. P., \& Kaya, A. (2014). Critical role of lay health cultural brokers in promoting the health of immigrants and refugees: A case study in the United States of America. International Journal of Human Sciences, 11(1), 291302. doi: $10.14687 /$ ijhs.v11i1.2723

\section{Introduction}

Although America is a nation which was founded and developed by immigrants, current statistics show that immigration is more prevalent now than in the past decades (Jiménez, 2011; Bailey, 2004; Active Voice, 2003; Portes \& Rumbaut, 1996 \& Rotich, 2009). Recent statistic from the US department of Homeland security indicates that, 58,179 persons were admitted to the United into the United States in 2012. Of these total, Bhutan, Burma, and Iraq had the greatest citizens represented. Additionally, 29,484 persons were granted asylum, 17,506 were granted asylum affirmatively, while 11,978 were granted asylum defensively by the Department of Justice. China, Egypt and Ethiopia had the greatest individuals who were admitted through the affirmative or defensive asylum.

Demographic reports from Active Voice show that, in 2000, the United States of America was home to 56 million foreign-born residents and children of immigrants, compared to 34 million that was reported three decades earlier. According to Jiménez, (2011), the United States is in the midst of another wave of mass immigration from Latin America (Mexico in particular), Asia, and the Caribbean. Active Voices (2003), also points out that these immigrants who are from different cultures, ethnicities, nationalities, and socio-economic status are settling and making new homes in urban and suburban cities across the Country. Additional reports from the US department of Homeland security show that 159 million nonimmigrant individuals were granted admission into the United States in 2012.

This increase in numbers of immigrants is reflected in almost all parts of the United States and particularly in North Carolina and Guilford County. Reports from the Faith Action International show that North Carolina and Guilford County have continued to experience a dramatic influx of immigrants since the early 1990s. According to Bailey, 2002; Rotich, 2009 \& Sills, 2002, statistics show that Guilford County witnessed over 50,000 immigrants over the last decade. More than 60 percent of the foreign-born immigrants entered the state of North Carolina and especially Guilford County during the last decade. The foreign-born population in North Carolina increased by 273.6 percent, growing from 115,077 to 430,000 residents between 1990 and 2000 (Piedmont Triad Council, 2002; \& Bailey, 2002). Guilford County resettles more refugees than any County in the entire state of North Carolina and has the largest and fastest growing numbers of immigrants of different nationalities, cultures, ethnicities, languages, religions, race, and ages.

Although the highest numbers of immigrants come from Mexico and other Hispanic/Latinospeaking regions, the first groups of immigrants in Guilford County are from the Northern continent of Europe. Other regions that are represented by the immigrants in Guilford County 
Rotich, J. P., \& Kaya, A. (2014). Critical role of lay health cultural brokers in promoting the health of immigrants and refugees: A case study in the United States of America. International Journal of Human Sciences, 11(1), 291302. doi: $10.14687 /$ ijhs.v11i1.2723

include Africa, Southeast Asia, South America, Eastern Europe, and the former Soviet Union (Sills, 2002, \& Bailey 2002). However, the total number of the African communities in Guilford County may not be a true representation because the mainstream system tends to identify or categorize them as African Americans. According to Bailey, the first African refugees were resettled in Guilford County in the early 1980s, and this first group was mainly from Ethiopia. Nevertheless, currently there are over 20,000 African immigrants in Guilford County representing various African nationalities. Though African immigrants continue to be invisible, the majority are attracted to the two historically black public universities in the Triad, North Carolina Agricultural \& Technical University in Greensboro and Winston-Salem State University in Winston-Salem (Sills, 2002; \& Bailey 2002). They work and serve collaboratively under the North Carolina African Service Coalition. The Latino immigrants, on the other hand, are the largest in the county as well as the state. Specific statistics shows that there are over 392,599 Hispanic or Latinos in North Carolina. The majority of these were born in Mexico (71\%); Puerto Rico (10\%), and Cuba (2.5\%). Another $16 \%$ is comprised of many other Latinos from central or South American countries. There are over 30,000 immigrants in Guilford County. They have different grass root nonprofit organization in Guilford County and across North Carolina.

Marshville, North Carolina, has the greatest number of Turkish Immigrants. According to AbdAllah, Umar Faruq (2010), the know Turkish settlers in America arrived in 1586 when Sir Francis Drake brought at least two hundred Muslims, identified as Turks and Moors, to the newly established English colony of Roanoke on the coast of present-day North Carolina.

Related statistics show that there are over 79,000 Southeast Asians in North Carolina, including a total population of over 7000 who have settled in Guilford County. The Southeast Asians have many grass root organizations in Guilford County namely: Montagnard Dega Association, Montagnard International Bible School, Vietnamese Temple, and Buddhist Temple. Most of these Southeast Asians consist of the Montagnards, Vietnamese, Cambodians, and Laotians. They are very different, and they represent different ethnicities, education levels, and religious identities.

However, regardless of their category, all immigrants and refugees have come and continue to arrive in the United States, North Carolina, and Guilford County with myriad hopes. They are fleeing poverty and persecution, pursuing athletic or educational glory, and seeking better and healthier ways of living. Most of them perceive America to be a beacon of hope and a final destination of refugee. The refugees and asylees, for instance, are a unique category of immigrants who are admitted into the United States because they are fleeing extreme persecution, war or natural disaster. Immigrants, on the other hand are in several states in the United States of America 
Rotich, J. P., \& Kaya, A. (2014). Critical role of lay health cultural brokers in promoting the health of immigrants and refugees: A case study in the United States of America. International Journal of Human Sciences, 11(1), 291302. doi: $10.14687 /$ ijhs.v11i1.2723

voluntarily in search of better ways of living. Unfortunately and unexpectedly, some of their hopes were thwarted upon arrival to their new land of "hope". Most of them have encountered some or all the following challenges.

\section{Immigrant and Refugee Barriers to Accessing Quality Health and Preventive Services}

Communication due to language and accent differences continues to play a pivotal and decisive role because it impedes access and compromises the quality of care that most immigrants and refugees receive (Ku \& Flores, 2005; \& Rotich, 2009). Most immigrants and refugees come from many countries and regions of the world and speak different languages and dialects other than English. For the vast majority, English language was neither their national nor their official language that was spoken in their home countries. Additionally, immigrants and refugees who are less proficient in the English language find it extremely difficult to communicate with their health care providers. This also makes it impossible to navigate the formal health care system. As a result, most of them end up utilizing the emergency room and home remedies as their primary sources of care. It is even more critical because most health and preventive care providers do not have access to a trained interpreter. Because of this communication barrier, most immigrants and refugees are less likely to: seek routine preventive health care, return for follow up appointments, finish their prescribed medications, and follow up with their medication instructions (Ku \& Flores, 2005; \& Rotich, 2009). The literature on access related barriers also indicates that some immigrants and refugees are illiterate in their own language. Reports in the Kaleidoscope indicate that the number of immigrants and refugees who are illiterate in their own languages is increasingly high (Kaleidoscope, 2003). Although some providers have made efforts to translate some materials into the different languages and dialects, it is still unfortunate because some immigrants and refugees find it difficult to read and write in their own language. Thus making it difficult to even read and understand any translated material such as medical forms, medical instructions, and instructions on medications among others.

Income and health insurance are crucial for entry into the formal health care system in the United States of America (Sudanno \& Baker, 2003). Due to socioeconomic hardships, a vast majority of immigrants and refugees in North Carolina were reported to be underinsured or uninsured, thus face substantial financial barriers to medical access. Most of them come from large families that are either inappropriately poor or work in low paying manual jobs that provide minimal or no health insurance. Coupled with the lack of work insurance, most of them have limited access to publicly funded programs such as Medicaid and Medicare. According to the provisions of the 1996-welfare reform law, undocumented immigrants are not eligible for standard benefits through Medicaid or 
Rotich, J. P., \& Kaya, A. (2014). Critical role of lay health cultural brokers in promoting the health of immigrants and refugees: A case study in the United States of America. International Journal of Human Sciences, 11(1), 291302. doi: $10.14687 /$ ijhs.v11i1.2723

the State Children's Health Insurance Program (SCHIP) and the regular Medicaid, and SCHIP benefits for the first five years they reside in the United States. Others (mostly refugees) have to wait for a period of five years before they could become eligible for most public benefits. Because of limited access to Medicaid or job-based health insurance, majority, especially the undocumented do not: access preventive care, receive early diagnosis or treatment, and follow through with appointments. A vast majority frequent the emergency room for all their primary care needs (Sudanno \& Baker, 2003; Bailey, 2002; Active Voice, 2003; Portes \& Rumbaut, 1996 \& Rotich, 2009).

Although the population of North Carolina is very diverse and has representatives from different backgrounds, it is unfortunate that the racial and ethnic makeup of the current health care workforce is not reflective of the state's ever increasing cultural diversity. This has mainly contributed to the underutilization of some medical services. This underrepresentation of immigrant and refugees in the health workforce makes it difficult for most immigrants to seek health care especially if their health concern is very sensitive. For one, they may not trust the provider because they are afraid that they may not understand their cultural views in regards to treatment and prevention. Secondly, they realize that it takes longer to get treatment and services because they have to involve an interpreter. On the contrary, they are known to frequent immigrant and refugee owned clinics or those agencies that have a diverse workforce. They seem to prefer the later because they understand their cultural views, perceptions, help seeking behaviors and dialects (Thom, Tirado, Woon, \& Mcbride, 2006).

Because of the language, cultural and transportation difficulties, most immigrants and refugees are uninformed and unaware of the available medical and preventive care services and programs. They have limited information on what is available, what they are eligible to receive, when the services are available and how to access them. As a result, most of them do not utilize the formal health care system or the preventive services and programs. Those who have tried to navigate the system have been turned off by the complicated protocol and the confusing eligibility requirements. Majority are unfamiliar with the bureaucracy, protocols and procedures such as making appointments, insurance requirements, paper work, time concept, payments, follow up and referrals among others. In addition, they do not receive timely and relevant information on prevention and treatment. The vast majority end up being diagnosed late and are less likely to receive timely preventive care or treatment. Additionally, the ongoing individual mobility that is very prevalent among most immigrant and refugee communities makes it difficult for them to become knowledgeable and familiar with the services available in their respective community. 
Rotich, J. P., \& Kaya, A. (2014). Critical role of lay health cultural brokers in promoting the health of immigrants and refugees: A case study in the United States of America. International Journal of Human Sciences, 11(1), 291302. doi: $10.14687 /$ ijhs.v11i1.2723

The impact of the increase in income inequality on the health and well-being of most immigrants and refugees continues to be significant. Due to low incomes, manual jobs, most of them are forced to settle in poorer and impoverished communities that do not offer health care resources and services (Bailey, 2004; Active Voice, 2003; Portes \& Rumbaut, 1996 \& Rotich, 2009). Because of the fear of the "unknown", most immigrants and refugees feel at ease living among their people. They, therefore, tend to gravitate towards areas and communities that have people who "look like them" and people who "speak like them. They form enclaves, which over time help contribute to the ever-occurring segregation. Because of this, most of them find themselves in neighborhoods that have limited health care resources. Generally, the more underserved the area is the greater the problems of access to health care due to geographic distances, and transportation problems.

Barriers to accessing health care are exacerbated by lack of legal documents. Families and individuals who are not documented do not freely seek health care services because they are constantly living in fear of deportation. As a result, some have chosen to stay away from such programs because of fear of immigration consequences. Others refrain from using public benefits because of the fear that seeking for services in the community or schools may create problems with the department of Home land Security and may subject them to deportation. This immigration related fears continue to be further compounded and complicated by the contentious politics that currently surround immigration. Those who have attempted to seek services outside of the emergency room have had difficulties understanding the protocols and the amount of time they have to wait before they can see a doctor. The vast majority of immigrants and refugees do not seek health care because they do not understand how to navigate the seemingly complicated system. Some immigrants especially those from developing countries feel that the very sophisticated technologies in the American health care system, deprives them of the hands-on examination and diagnosis. As the research further indicates, the majorities of the newcomer immigrants are low income and have limited knowledge of western industrial concepts of health and wellness practices. These challenges are more profound among the Latino, African, and Southeast Asian communitiesthe predominant immigrant populations. The smaller local European and Eastern European communities, less than 5,000 people, are more familiar with western scientific and technological concepts and their cultures more closely parallel western society. Health literacy and knowledge of western concepts can result in profound health problems among immigrants and refugees.

Cultural differences and conflicts remain a significant challenge to most newcomers. Ethnically distinct cultural practices, beliefs and perceptions of health care, healthy lifestyles and roles of each family member may conflict with the western views. Most immigrants and refugees encounter 
Rotich, J. P., \& Kaya, A. (2014). Critical role of lay health cultural brokers in promoting the health of immigrants and refugees: A case study in the United States of America. International Journal of Human Sciences, 11(1), 291302. doi: $10.14687 /$ ijhs.v11i1.2723

diverse cultural conflicts as they strive to navigate the health care system and as they make decisions to seek help in their new environment. According to Bailey, some of the challenges include cultural and generational conflicts, lack of culturally appropriate resources, and especially the challenge of transforming cultural traditions or developing new cultural paradigms that provide healthy lifestyles suitable for this culture. Unlike in the western culture, the decisions of all family elders and community members as to when to seek healthcare takes precedence over follow up appointments. This process may deter individuals from follow up appointments or preventive health care measures.

Due to experience, immigrants and refugees take time before they can discuss their problems openly with providers (Bailey, 2004; Active Voice, 2003; Portes \& Rumbaut, 1996 \& Rotich, 2009). They particularly felt uncomfortable with the presence of interpreters and with the presumed intrusive and personal questions that their health care providers often asked.

\section{Lay Health Cultural Brokers}

Notwithstanding the pivotal role of immigrants and refugees in the United States and North Carolina, it unfortunate because the above evidence portray critical acculturation challenges that call for an immediate collaboration consideration. One way to address this is to actively engage the lay health cultural brokers. According to Hoge, Marrelli, \& Tondora (2005), Lay Health advisors are informal cultural advisors who give or natural cross-cultural helpers. They are cultural change agents who provide intervention programs that help to link their communities to the health care system.

Most lay health advisors self identified or were identified by the leaders from neighborhood associations and community-based organizations. In an effort to outline innovative interventions and resources that can help address the health and acculturation challenges of immigrants and refugees, the purpose of this case study was to examine the specific roles of lay health cultural brokers.

\section{Methodology}

The participants for this case study were 10 Lay health cultural brokers ( 5 male and 5 female). The ten were recruited based on the following criteria: The ten were recruited based on the following criteria: (1) immigrant and refugee, (2) lay health cultural brokers serving immigrant and refugee communities, (3) resident of North Carolina, (4) and had served as a lay health cultural broker for a minimum of one year. The qualitative data was collected through focus group and one-on-one indepth interviews. The in-depth interviews brought out some details on their day to day activities 
Rotich, J. P., \& Kaya, A. (2014). Critical role of lay health cultural brokers in promoting the health of immigrants and refugees: A case study in the United States of America. International Journal of Human Sciences, 11(1), 291302. doi: $10.14687 /$ ijhs.v11i1.2723

(Kitchin and Tate, 2000). The interviews considered several questions concerning their daily roles (e.g., what services to you provide for the immigrants and the service providers/)

Participant Selection: Criterion- based sampling (Goetz \& LeCompte, 1984) was used to select the participants for this study. The researcher used community leaders from different immigrant and refugee communities to recommend and recruit a convenient sample based on the set criteria. They recommended and contacted a chain of individuals in their community who would be good sources of information and who would make a commitment to participate in one-on-one and focus group interviews.

\section{Research Design}

Focus groups and one-on-one interviews were used to collect the qualitative data for this study. They were asked to describe their roles as lay health advisors.

\section{Results}

The qualitative case study elicited valuable information regarding the roles of lay health cultural advisors. The lay health cultural brokers engaged in: (1) helping immigrants and refugees understand the health care system, (2) providing interpreter and translator services, (3) providing preventive educational programs, and (4) providing transportation. They also organized and facilitated cultural-competency trainings for various health care providers. Detailed descriptions of the findings are described below. When the lay health advisors were asked to explain what they do, eleven primary roles emerged. The next sections highlight details of these roles. The lay health advisors indicated that they:

\section{1) Provide and Facilitate Cross Cultural Competency Trainings:}

Lay health cultural brokers reported that they played a vital role in planning and facilitating multifaceted, multicultural, multisite and collaborative cross cultural competency trainings in the community, schools, health care systems and the cooperate world. Most of their trainings were aimed at helping the health care and preventive health professionals understand the needs and challenges of the various immigrants and refugees, thus equipping them with necessary essential knowledge, skills and tips on how to provide culturally sensitive services to their diverse clientele. These trainings also helped providers to be better prepared to give more quality health care and preventive healthy lifestyle programs for many newcomer communities. The lay health advisors pointed out that, most providers were better equipped to serve many immigrants and refugees if they had some background information on their prior experiences and current acculturation challenges. 
Rotich, J. P., \& Kaya, A. (2014). Critical role of lay health cultural brokers in promoting the health of immigrants and refugees: A case study in the United States of America. International Journal of Human Sciences, 11(1), 291302. doi: $10.14687 /$ ijhs.v11i1.2723

2) Develop Training Materials: Lay health cultural brokers indicated that they developed training resources and materials that were made available to providers who serve immigrants and refugees. These materials were made available either through print, or online. They gave an example of the "Kaleidoscope". This unique training manual was developed and published by the cultural competency task force that comprised of various stakeholders and agencies from the county. This resource guide was distributed to all health care providers in the county. They indicated that the availability of related resource materials would be extremely beneficial to all service providers.

3)Provide a Road Map of Services: The lay health cultural brokers also reported that they provided educational information to the immigrant and refugee community leaders and representative on: (1) how to navigate the health care systems, (2) health care protocols, (3) and the requirements and details on eligibility requirements for various services. They alluded to the fact that this was crucial because it helped to demystify the otherwise seemingly complex healthcare systems related difficulties.

4) Provide Interpreter Services: They served as bilingual Interpreters for health care providers among other professional services. According to Fatima, her refugee client from Sierra Leone, noted that her elderly parents who were forced to leave their country after the civil war in the 1990's, had a difficult time accessing health care because they were unable to speak English or French. She indicated that her parents only spoke their local language Mende and Kriol. Maria, an immigrant from Mexico, who only spoke Spanish and H'Bong a refugee from Vietnam, who spoke Vietnamese and Jarai, also encountered similar communication barriers. Halima a lay Health Advisor from Somali also alluded to this. She narrated what her client went through after she had surgery. She noted that she was not able to go back for follow up routine examinations after surgery because she was unable to get an interpreter who spoke her language

5) Translate Materials: Lay health cultural brokers also assist providers by translating vital materials in as many languages and dialects as possible. They indicated that, although efforts have been made to translate health care materials such as forms, side effects of medications, eligibility guidelines among many others, they are mainly in Spanish and not in all other represented languages.

6) Contribute to the Diverse Workforce: They all indicated they were among the few culturally and linguistically diverse workforces in the health field. They, however, pointed out that, there was a significant need to encourage and to train more diverse cultural brokers to apply for jobs in the health care systems and environments. Morales, a lay health advisor from Mexico and Mai, a refugee from Cambodia, echoed that their clients preferred clinics and health care offices that hired 
Rotich, J. P., \& Kaya, A. (2014). Critical role of lay health cultural brokers in promoting the health of immigrants and refugees: A case study in the United States of America. International Journal of Human Sciences, 11(1), 291302. doi: $10.14687 /$ ijhs.v11i1.2723

people who looked like them and those who understand their cultures and language. This diversity in the workforce benefited the immigrant and refugee community as well as the health care system.

7) Provide Mentorships: Lay health cultural brokers indicated that they served as mentors to younger and newly arrived immigrants and refugees in many different ways. They pointed out that, the teenagers and college age students came to them for guidance because they were struggling to fit into the American culture as well as maintain their original traditions. They indicated that mentoring was a high acculturation intervention because their services expanded way beyond the healthcare setting.

8) Represented their Communities: They indicated that they represented their communities in local coalitions and collaborations as well as kept them informed on any changes or current events and issues. They noted that ongoing collaborations between health care providers and many immigrant and refugee stakeholders helped to coordinate and enhance existing services to ensure that all communities were receiving quality services.

9) Guide Health Care systems to Make the Environments Culturally Inviting: Lay health cultural brokers provided ongoing input, information and feedback on how health care systems can create culturally inviting and sensitive health care environments (i.e. directions and signs in different languages, use of pictures and signs, culturally competent front line workers etc).

10) Provide Education: They provided community-wide comprehensive awareness and education through workshops, radio or television programs. They were engaged in educating, motivating, referring and encouraging community members to incorporate healthy lifestyles that would "improve their health and overall quality of life".

11) Provide on-Going Acculturation Trainings for Immigrants and Refugees: Halima pointed out 'Such trainings enhanced awareness and knowledge on available resources, how to navigate the health care system; service eligibility; and positive, healthy lifestyle practices". They also provided a great opportunity for many immigrants to share experiences and exchange ideas. Additional, they helped to build cohesive groups that were enthusiastic and energized to serve their communities.

Their significant roles are a clear indication of the urgent and critical need to engage more lay health cultural brokers and community leaders from many immigrant and refugee communities. Their services will continue to support health and preventive care professionals to recognize: (1) the context in which particular behaviors occur; (2) the nature of reactions and adaptations; (3) the direct relationship to the socioeconomic status; (4) the cultural, regional and gender differences; (5) the social injustices; (6) and the situation and language constraints that often affect their health and health seeking behaviors. They helped providers to be better equipped to serve their many immigrant and refugee clients. 
Rotich, J. P., \& Kaya, A. (2014). Critical role of lay health cultural brokers in promoting the health of immigrants and refugees: A case study in the United States of America. International Journal of Human Sciences, 11(1), 291302. doi: $10.14687 /$ ijhs.v11i1.2723

\section{Conclusions}

Given the myriad challenges that immigrants and refugees face, it is apparent that lay health cultural brokers can provide an effective alternative to enhancing the health and the acculturation process of many immigrants and refugees. It is also apparent that the ever-increasing conflicts and calamities in different parts of the world necessitate a closer attention to cultural competency and the role of cultural brokers. Under these circumstances, it is evident that there is a need to utilize the expertise of various cultural brokers such as lay health advisors because they are better placed to communicate the nuances, needs and values of the different individuals to the mainstream American community.

\section{References}

Abd-Allah, Umar Faruq (2010), Turks, Moors, \&Moriscos in Early America: Sir Francis Drakes' Liberated Galley Slaves \& the Lost Colony of Roanoke.

Active Voice, (2003). The New Americans. \{Information posted on the website\}. Retrieved April 2013 from the web: http://www.activevoice.net

Bailey, R. (2004). Demographics of Immigrants in Guilford County, NC. bttp:// cnnc.uncs.edu/information/pop_demgraphics.html

Bailey, R. (2002). Demographics of Immigrants in Guilford County, NC. \{Report posted on the Web\}.Retrieved May 2011, from the CNNC web: bttp:/ / cnnc.uncg.edu/information/pop_demgraphics.html

Balgopal, R.P., (2000). Social work practice with immigrants and refugees. Columbia University Press: New York.

Goetz, J.E., \& LeCompte, M.D (1994). Ethnography and qualitative design in Educational Research. Orlando, FL: Academic Press.

Hoge, M., Marrelli, A., \& Tondora, J. (2005). The fundamentals of workforce competency: implications for behavioral health. Administration and Policy in Mental Health, 32(5-6), 509-531.

Jiménez, Tomás R. (2011). Immigrants in the United States: How Well Are They Integrating into Society? Washington, DC: Migration Policy Institute.

Kaleidoscope, (2003). Cultural Diversity in Guilford County. A training handbook for Health and human service providers.

Ku L., Flores G..., (2005). Pay Now or Pay Later: Providing Interpreter Services in Health Care: Helping the Millions of Americans with Limited English Proficiency Can Improve the Quality of Care and Reduce the Risk of Medical Errors. Health Affairs. 24:435-444

Kitchin, R. and Tate, N. (2000). Conducting Research into Human Geography: Theory, Methodology, and Practice. Essex-England: Pearson Education Limited.

Lacere, F., Jensen, L., \& Biddlecom. A. (1994). Health Care Utilization, Family Context and Adaptation among Immigrants to the United States. Journal of Health \& Social Behavior 35 (4), 370-384

Portes, A., \& Rumbaut, R.G. (1996). Immigrant America, Berkeley, CA: University of California Press. Piedmont Triad Council of Governments. (2000). "Census 200 Data." Piedmont Triad Council of Governments. http://www.ptcog.org/. Piedmont Triad Council of Governments, Piedmont Triad, http://www.faithaction.com/.

Sills, M. (2002). "2002 Annual Report on the Hispanic/Latino Population in all 100 North Carolina Counties." Faith Action International House. 
Rotich, J. P., \& Kaya, A. (2014). Critical role of lay health cultural brokers in promoting the health of immigrants and refugees: A case study in the United States of America. International Journal of Human Sciences, 11(1), 291302. doi: $10.14687 /$ ijhs.v11i1.2723

Rotich, J.P. (2009). Cross Cultural Competency: Providing Cross Culturally Appropriate Health Care Services and Environments to Diverse Immigrants and Refugees. In R. Staudinger, H. Ostermann \& B. Staudinger (Eds.), Handbook of Research in Nursing Informatics and Socio technical Structures: Information Science Reference, IGI Global.

Sills, M. (2002). "2002 Annual Report on the Hispanic/Latino Population in all 100 North Carolina Counties." Faith Action International House.

Sudanno, J.J; \& Baker, D.W, (2003). Intermittent lack of Insurance Coverage and use of preventive services. American Journal of public Health. 93:130-137.

Thom, D., Tirado, M., Woon, T., \& Mcbride, M. (2006). Development and evaluation of a cultural competency-training curriculum. BMC Medical Education, 6, doi: 10.1186/1472-6920-6-38.U.S. Department of Homeland Security, Customs and Border Protection (CBP), Operations Management Reporting, Fiscal Year 2011. 